\title{
Selective Excitation of Harmonics in a Collisionless Plasma by Two Counterstreaming Electron Beams
}

\author{
W. L. Sadowski and Z. G. Ruthberg \\ Institute for Basic Standards, National Bureau of Standards, Washington, D.C. 20234
}

(August 22, 1969)

\begin{abstract}
The possibility of selective harmonic excitation in a one-dimensional plasma that obeys the Vlasov equation is discussed. Electron beams, collimated in velocity space, are used for this purpose. Conditions on the velocity distribution function to achieve selective excitation are given. The velocity distribution function is expanded in Gram-Charlier series and the expansion coefficients are given. Bounds on truncation errors of the expansion are derived and compared with computer results.
\end{abstract}

Key words: Electron trapping; non-linear; numerical; selective harmonic excitation; two-stream; velocity distribution; Vlasov equation.

\section{Introduction}

This paper deals with the feasibility of a numerical study of higher harmonic excitation of the non-linear Vlasov plasma by two counterstreaming electron beams. In section 1 it is shown that an electron beam with a high degree of collimation in velocity space can couple to the phase velocity of one of the higher harmonics. The coupling should provide preferential excitation of the harmonic. The collimation is achieved by expressing the velocity distribution function in the form $\exp \left[-(v \pm a)^{2} / 2 \epsilon\right]$ with $\epsilon$ being the collimation parameter that determines the halfwidth of the velocity distribution curve. The distribution function is expanded in Gram-Charlier series and the expansion coefficients are derived in section 2. The necessary transformations of Hermite polynomials are given in the paper "Temperature Renormalization in the Non-Linear Vlasov Problem," immediately preceding this paper. In section 3 are discussed convergence properties of the Gram-Charlier expansion and a criterion is given that allows one to calculate an upper bound on the number of terms necessary to represent the initial distribution function to the desired degree of accuracy.

Comparison of analytic results with those obtained on the computer are discussed in the appendix.

\section{Selective Excitation of Harmonics}

Numerical work on the solution of the non-linear Vlasov equation by the method of eigenfunction expansion performed by one of the authors [1] ${ }^{1}$ brought out some interesting facts associated with the non-linear character of the equation. One of these is that the ratio of plasma frequencies of successive harmonics is not an integer. For example for $k=0.5$

$$
\omega_{2} / \omega_{1}=1.86
$$

${ }^{1}$ Figures in brackets indicate the literature references at the end of this paper. 
where $k$ is the wave number of the fundamental, $k=2 \pi / L$. The phase velocity of a standing wave in a plasma is given by

$$
v_{p h}=\frac{\omega_{p}}{k}
$$

with $\omega_{p}$ being the plasma frequency. Since the ratio of $k$ 's in a periodic system is an integer, we can see from eqs (1.1) and (1.2) that the phase velocities of the fundamental and the second harmonic do not coincide. In the example quoted above the phase velocities of the fundamental and the second harmonic are respectively

$$
v_{p h 1}=2.71 \quad \text { and } \quad v_{p h 2}=2.52 .
$$

The difference in phase velocities is $\Delta v=0.19$. This fact suggests the possibility of a computer study of selective excitation of harmonics by two counterstreaming beams of electrons traveling through the plasma. The electron beams must be sufficiently well collimated in velocity space so as to couple to the desired harmonic. The amount of trapping as a function of the distance from the phase velocity was given by J. Dawson [4]. The functional dependence is rather complicated and will not be given here. It is simpler to use numerical results to examine the possibility of selective excitation of harmonics. Figure 1 shows $f(v)$ that satisfies the non-linear Vlasov equation ${ }^{2}$ for $k=0.5, \alpha=0.1$ at the dimensionless time $t=32$.

The amplitude of the second harmonic was at all times at least an order of magnitude less than that of the fundamental. The width of the shoulder as can be seen from the insert in figure 1 is about 1.54, with the phase velocities of the fundamental and the second harmonic located on this shoulder. Thus if an electron beam with a velocity distribution with a halfwidth of the order of separation of the two phase velocities were centered on the phase velocity of the second harmonic, the interaction of the second harmonic with the electron beam would excite this harmonic. The effect of the beam on the fundamental would be much less pronounced because of the lower density at the phase velocity of the fundamental.

Since the initial distribution function [1] was chosen to be Maxwellian, a natural choice for the two-stream distribution function is also a Maxwellian plus a combination of Maxwellians centered at $a$ and $-a$.

$$
f(v, t=0)=\frac{1}{\sqrt{2 \pi}(1+2 \gamma \sqrt{\epsilon})}\left[e^{-\frac{v 2}{2}}+\gamma e^{-\frac{(v-a) 2}{2 \epsilon}}+\gamma e^{-\frac{(v+a) 2}{2 \epsilon}}\right]
$$

where $\frac{1}{\sqrt{2 \pi}(1+2 \gamma \sqrt{\epsilon})}$ is the normalization constant. The parameter $\epsilon$ determines the halfwidth of the two-stream distribution function. To obtain a halfwidth of $\Delta v, \epsilon$ must satisfy the relation

$$
\frac{(\Delta v)^{2}}{2 \epsilon}=1
$$

For $\Delta v=0.19$ this gives the value

$$
\epsilon=1.81 \times 10^{-2} \text {. }
$$

\footnotetext{
${ }^{2}$ Unpublished results
} 
The individual components of the two-stream distribution functions are expressed in the form

$$
\begin{gathered}
e^{-\frac{(v-a) 2}{2 \epsilon}}=\sum_{n} C_{n} H e_{n}(v) e^{-\frac{v^{2}}{2}} \\
e^{-\frac{(v+a) 2}{2 \epsilon}}=\sum_{n} D_{n} H e_{n}(v) e^{-\frac{v^{2}}{2}}
\end{gathered}
$$

where $\mathrm{He}_{n}$ are Hermite polynomials. The convergence of the expansion, the value of its coefficients, and the number of terms in the expansion will be treated in detail below.

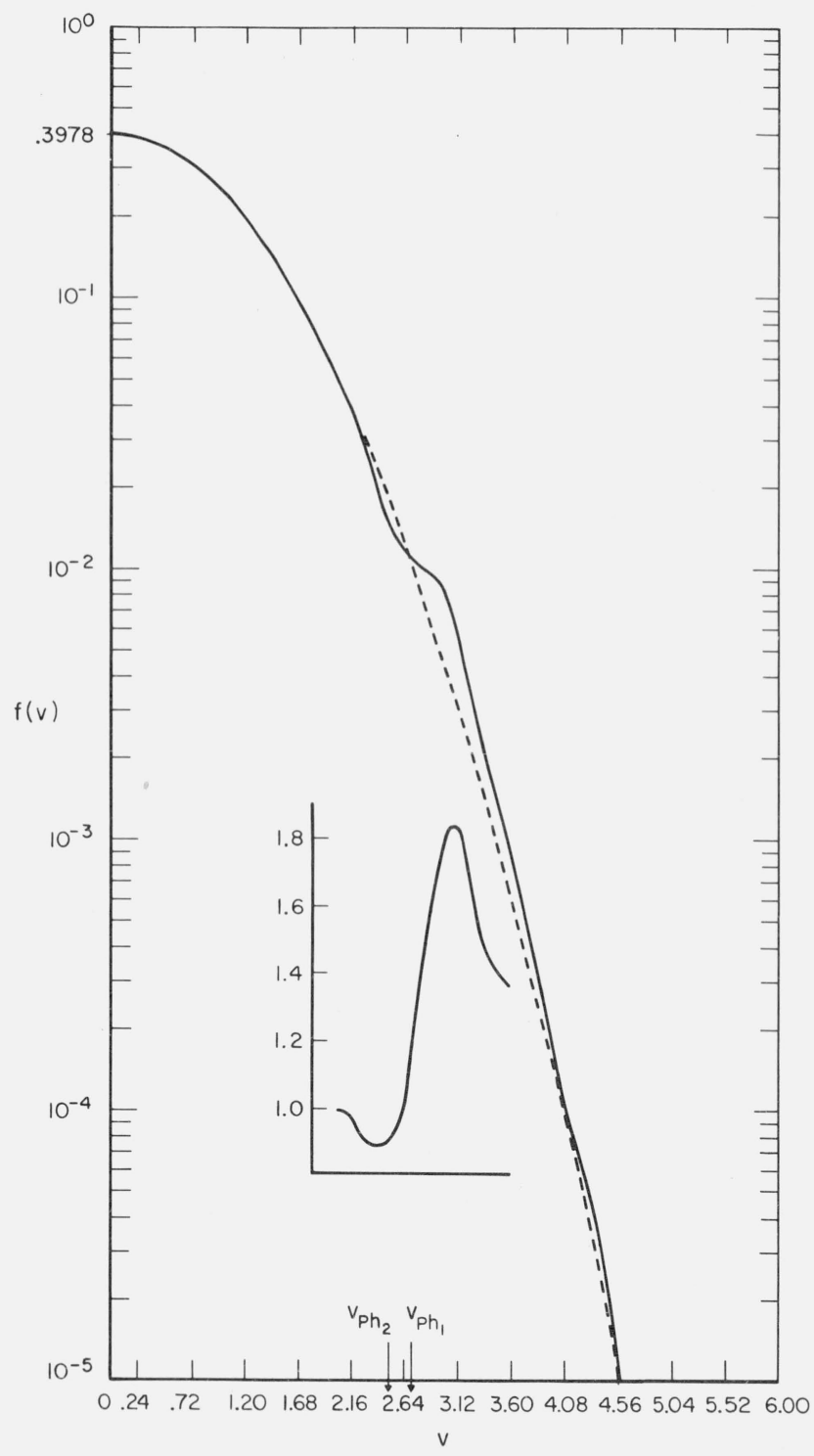

FiguRE 1. $\mathrm{f}(\mathrm{v})$ versus $\mathrm{v}$ for $\mathrm{k}=0.5$ at $\mathrm{t}=32$.

The dashed line gives $e^{-v^{2} / 2} / \sqrt{2 \pi}$ for comparison. The insert represents $f(v) /\left(e^{-v^{2} / 2} / \sqrt{2 \pi}\right)$. 


\section{Expansion Coefficients for the Two-Stream Problem}

We want to express one of the side peaks of the two-stream velocity distribution function of eq (1.5) in the following form:

$$
e^{-\frac{(v-a) 2}{2 \epsilon}}=g(v) e^{-\frac{v 2}{2}}
$$

where

$$
g(v)=\sum_{n=0}^{\infty} C_{n} \mathrm{He}_{n}(v)
$$

Coefficients for the other side peak can be obtained immediately by substituting $a$ for $-a$, as can be easily seen by comparing eqs TR $(1.14)^{3}$ and TR (1.15). The expansion coefficients, $C_{n}$, are given by the integral:

$$
C_{n}=\frac{1}{\sqrt{2 \pi} n !} \int_{-\infty}^{\infty} g(v) e^{-\frac{v^{2}}{2}} H e_{n}(v) d v .
$$

Substituting the expression for $g(v)$ from eq $(2.1)$, we obtain

$$
C_{n}=\frac{1}{\sqrt{2 \pi} n !} \int_{-\infty}^{\infty} e^{-\frac{(v-a) 2}{2 \epsilon}} H e_{n}(v) d v .
$$

Let us now make the transformation

$$
\bar{v}=\frac{v-a}{\sqrt{\epsilon}} .
$$

This leads to the integral

$$
C_{n}=\frac{1}{\sqrt{2 \pi} n !} \frac{1}{\beta^{1 / 2}} \int_{-\infty}^{\infty} e^{-\bar{v}^{2} / 2} H e_{n}\left(\frac{\bar{v}+\bar{a}}{\sqrt{\beta}}\right) d \bar{v} .
$$

Here $\beta=\epsilon^{-1}$ was introduced to express the argument of $H e_{n}$ in the form given in eq TR (1.15), and $\bar{a}=\frac{a}{\sqrt{\epsilon}}$. Since $H e_{0}=1$ the integrad in eq (2.6) can be considered as a product

$$
H e_{n}=H e_{0} H e_{n}
$$

$H e_{n}\left(\frac{\bar{v}+\bar{a}}{\sqrt{\beta}}\right)$ is expressed in terms of $\sum H e_{n}(\bar{v})$ by eq TR (1.15). From the orthogonality conditions we see that the only term in the sum that contributes to the integral is the term with $p=n$.

Substituting this term for $H e_{n}\left(\frac{\bar{v}+\bar{a}}{\sqrt{\beta}}\right)$, integration can be carried out and we obtain:

$$
C_{n}=\frac{1}{n !} \epsilon^{1 / 2}(1-\epsilon)^{n / 2} H e_{n}\left(\frac{a}{\sqrt{1-\epsilon}}\right)
$$

${ }^{3}$ TR-refers to "Temperature Renormalization in the Non-Linear Vlasov Problem" by the same authors, immediately preceding this paper. 
Substituting the values of $C_{n}$ obtained in eq (2.8) into eq (2.2), we can now express eq (1.3) in terms of its Hermite polynomial expansion.

$$
\begin{aligned}
f(v, t=0)= & \frac{e^{-v^{2} / 2}}{\sqrt{2 \pi}(1+2 \gamma \sqrt{\epsilon})}\left[1+\gamma \sum_{n=0}^{\infty} \frac{\epsilon^{1 / 2}(1-\epsilon)^{n / 2}}{n !} H e_{n}\left(\frac{a}{\sqrt{1-\epsilon}}\right) H e_{n}(v)\right. \\
& \left.+\gamma \sum_{n=0}^{\infty} \frac{\epsilon^{1 / 2}(1-\epsilon)^{n / 2}}{n !} H e_{n}\left(\frac{-a}{\sqrt{1-\epsilon}}\right) H e_{n}(v)\right] .
\end{aligned}
$$

Since Hermite polynomials with odd indices are odd functions, eq (2.9) reduces to its final form

$$
f(v, t=0)=\frac{e^{-v^{2} / 2}}{\sqrt{2 \pi}(1+2 \gamma \sqrt{\epsilon})}\left[1+2 \gamma \sum_{n=0}^{\infty} \frac{\epsilon^{1 / 2}(1-\epsilon)^{n}}{(2 n) !} H e_{2 n}\left(\frac{a}{\sqrt{1-\epsilon}}\right) H e_{2 n}(v)\right]
$$

\section{Convergence Properties of the Expansion}

In numerical work it is desirable to truncate the expansion after the smallest number of terms for a given truncation error. Using the asymptotic form for Hermite polynomials [6] (see TR appendix I for the discussion)

$$
H e_{n}(x)=\frac{e^{x^{2 / 4}}}{2^{n / 2}} \frac{\Gamma(n+1)}{\Gamma\left(\frac{1}{2} n+1\right)}\left[\cos \left(N^{1 / 2} \frac{x}{\sqrt{2}}-\frac{1}{2} n \pi\right)+0\left(n^{-1 / 2}\right)\right]
$$

where $N=2 n+1$. For large $n$, we obtain the asymptotic expression for

$$
C_{n} H e_{n}(v) \sim \frac{\epsilon^{\frac{1}{2}}}{n !} \frac{(1-\epsilon)^{\frac{n}{2}}}{2^{n}} e^{\frac{v^{2}}{4}+\frac{a^{2}}{4(1-\epsilon)}} \frac{\Gamma^{2}(n+1)}{\Gamma^{2}\left(\frac{1}{2} n+1\right)}
$$

with $C_{n}$ defined by eq (2.6).

In the above expression the term $0\left(n^{-1 / 2}\right)$ has been neglected and $\cos \left(N^{1 / 2} \frac{v}{\sqrt{2}}-\frac{1}{2} n \pi\right)$ has been replaced by unity.

Using the well-known relation between the $\Gamma$-function and the factorial

$$
\Gamma(z+1)=z !
$$

we obtain the asymptotic expansion for the terms in eq (2.10)

$$
C_{2 n} H e_{2 n}(v) \sim e^{K} \frac{\epsilon^{1 / 2}}{2^{2 n}} \frac{(2 n) !}{(n !)^{2}}(1-\epsilon)^{n}
$$

where $K=\frac{v^{2}}{4}+\frac{a^{2}}{4(1-\epsilon)}$. Using Stirling's formula for the factorials, we finally get:

$$
C_{2 n} H e_{2 n} \sim \frac{\epsilon^{1 / 2} e^{K}}{\sqrt{\pi}} \frac{(1-\epsilon)^{n}}{\sqrt{n}} .
$$

The asymptotic expression for $C_{2 n} H e_{2 n}$ in eq (3.5) tends monotonically to zero with increasing $n$. This can be shown easily by taking the logarithm of $\mathrm{C}_{2 n} \mathrm{He}_{2 n}$

$$
\ln C_{2 n} H e_{2 n} \sim \ln B+n \ln (1-\epsilon)-\frac{1}{2} \ln n
$$


where

$$
B=\frac{\epsilon^{1 / 2} e^{K}}{\sqrt{\pi}}
$$

Since $0<\epsilon \leqslant 1, \ln (1-\epsilon)$ is a negative number and the logarithmic terms in eq (3.6) go to $-\infty$.

Thus

$$
\lim _{n \rightarrow \infty} C_{2 n} \mathrm{He}_{2 n}=0 \text {. }
$$

Let us now find the truncation error introduced into the function by neglecting all the terms in eq (2.10) past the term $H e_{2 N}$. This error can be represented in the form:

$$
R_{N+1}<\theta \frac{\epsilon^{1 / 2}(1-\epsilon)^{N+1}}{(2 N+2) !} H e_{2 N+2}\left(\frac{a}{\sqrt{1-\epsilon}}\right) H e_{2 N+2}(v) \sum_{k=0}^{\infty}(1-\epsilon)^{k}
$$

where

$$
\theta=2 / \sqrt{2 \pi}(1+2 \gamma \sqrt{\epsilon})
$$

Expressing all $\mathrm{He}_{2 N+2+p}, p=0$ to $\infty$ in their asymptotic form we see that since $1-\epsilon<1$, the geometric progression in eq (3.8) can be summed and we finally obtain a bound on the truncation error

$$
R_{N+1}<\theta \frac{e^{\frac{v^{2}}{4}+\frac{a_{2}}{4(1-\epsilon)}}(1-\epsilon)^{N+1}}{\sqrt{\pi} \epsilon^{1 / 2}(N+1)^{1 / 2}} .
$$

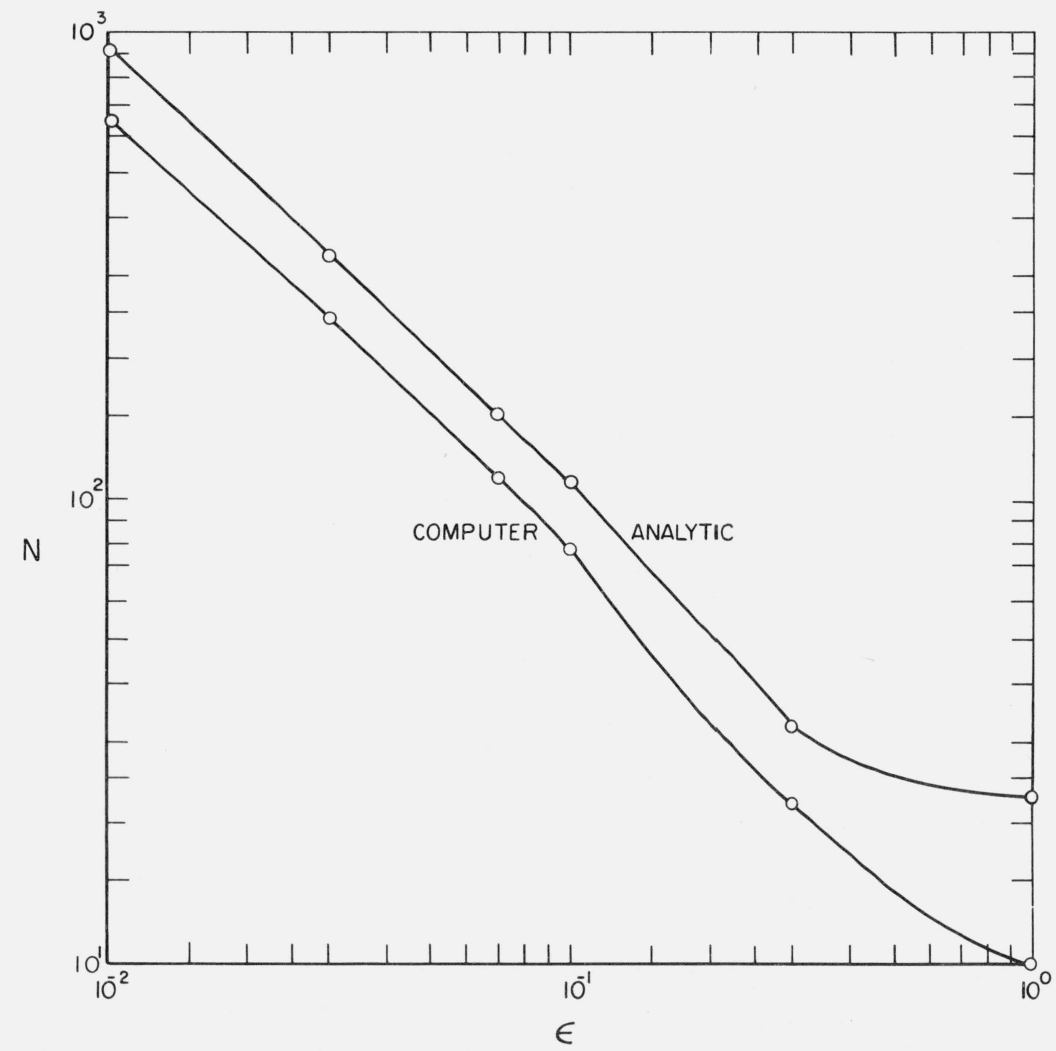

FIGURE 2. Nversus $\epsilon$ for two-stream function.

Computer results and analytic results for $v=3.0, a=2.0$ 
The inequality in eq (3.9) makes it possible to calculate $N$ for a given truncation error. This is useful in representing the initial two-stream distribution function in the Vlasov equation. Figure 2 gives the number of terms in the expansion of the expression given in eq (2.10) as a function of $\epsilon$ for $a=2.0$ and $v=3.0$ for a truncation error of $R_{n}<10^{-5} S$ (see appendix). A graph of the number of terms obtained on the computer is included for comparison. The values of $N$ for $\epsilon=0.9$ and 0.8 do not lie on the curve, which is not surprising since the asymptotic expression for $\mathrm{He}_{n}$ is not very accurate for $N \sim 10$. As $N$ increases the points form a curve which bounds from above the computed curve. This again is not surprising, since the approximations made in eqs (3.2) and (3.8) made the truncation error somewhat bigger than it actually is.

\section{Appendix}

A computer program was written in Fortran to calculate the terms of the expansion of $f(v, t=0)$ of eq (2.10). The $H e_{2 n}(v)$ and $C_{2 n}$ were calculated recursively. Scaled polynomials, $\bar{H} e_{2 n}$, were used to stay within the range of the computer in single precision.

$$
\begin{gathered}
\bar{H} e_{2 n}(v)=\frac{1}{\sqrt{(2 n) !}} H e_{2 n}(v) \\
\bar{C}_{2 n}=\sqrt{(2 n) !} C_{2 n} .
\end{gathered}
$$

A partial sum, $P_{n}$, of the first $n$ terms was formed and more terms were added until the relative error ratio test of eq (A.2) was satisfied for one hundred successive terms.

$$
\mid \text { R.E. }\left(P_{n}\right) \mid=\frac{\left|P_{n+1}-P_{n}\right|}{\left|P_{n}\right|}<10^{-5} \text {. }
$$

Using eqs (2.10), (3.5), (3.9), and (A.2), the relation of this ratio test to the truncation error is given by eq. (A.3).

where

$$
R_{N+1}<\frac{\theta e^{K}(1-\epsilon)^{N+1}}{\epsilon^{1 / 2} \sqrt{\pi}(N+1)^{1 / 2}}<10^{-5} S
$$

$$
S=\frac{\theta}{\epsilon} \frac{f e}{2}^{v^{2} / 2}
$$

and $f$ is the un-normalized function calculated on the computer. Figure 2 contains the computer results for $v=3.0, a=2.0$ and a comparison analytic curve based on eq (A.3). Other values of the parameters investigated on the computer were

$$
\begin{aligned}
\epsilon & =1.00,0.30,0.10,0.07,0.03,0.01 \\
a & =1.00,2.00,3.00,4.00,5.00 \\
v & =0.00 \text { to } 6.00 \text { in intervals of } 0.25 \\
\gamma & =0.1 .
\end{aligned}
$$

Figure 3 contains a set of results for $v=3.0, a=1.0,3.0,5.0$. 


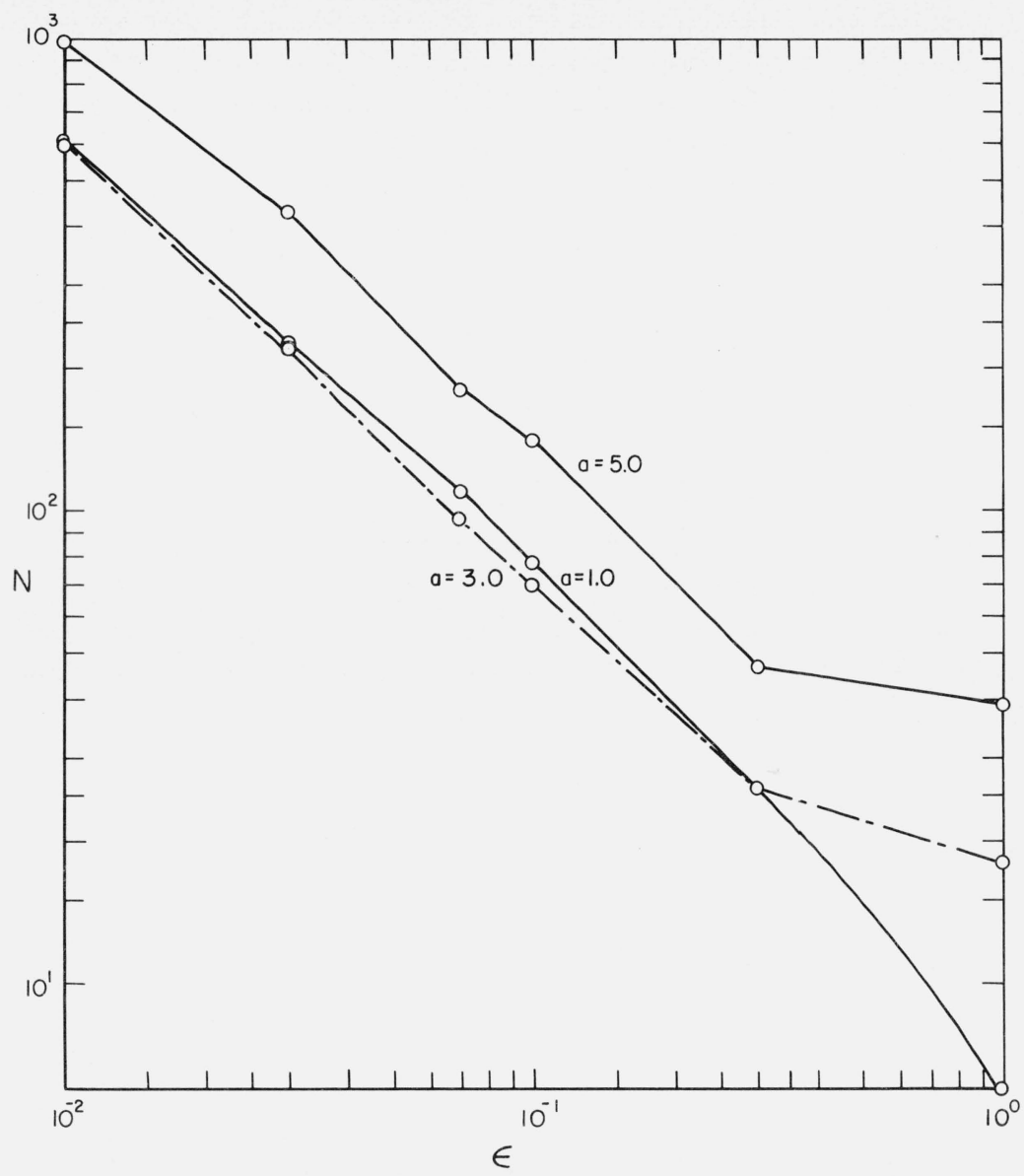

FiguRE 3. N versus $\epsilon$ for two-stream function.

Computer results for $v=3.0$

\section{References}

[1] Sadowski, W. L., On Some Aspects of the Eigenfunction Expansion of the Solution of the Non-Linear Vlasov Equation, Symposium on Computer Simulation of Plasma and Many-Body Problems, NASA,'SP-153, 433-440, (1967).

[2] Armstrong, T. P., Numerical Studies of the Non-Linear Vlasov Equation, Ph.D. Thesis, Univ. of Iowa, No. 66-34, (1966).

[3] Feix, M., Engleman, F., Minardi, E., and Oxenius, J., Nonlinear effects from Vlasov's equations, Phys. Fluids 6, 266$275,(1963)$.

[4] Dawson, J., On Landau damping, Phys. Fluids 4, 869-874, (1961).

[5] Knorr, G., Numerische Integration der nichtlinearen Vlasov Gleichung, Z. Naturforsch 16a, 1320-1328, (1961).

[6] Bateman Manuscript Project, Cal. Inst. Technol., Higher Transcendental Functions, A. Erdelyi, editor, Vol. 2, 201 (McGraw-Hill Book Co., N.Y., 1953). 